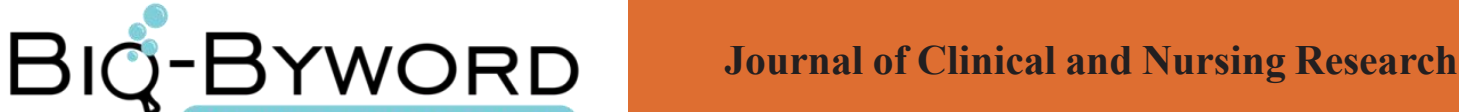

\section{Application of Two Different Monitoring in Blood Purification Capacity Management}

Fu Yanping, Fu Liqin, Yang Xianghong

Zhejiang Provincial People’s Hospital, Hangzhou, Zhejiang 310014, China

\section{ARTICLE INFO}

Article history:

Published online: $30^{\text {th }}$ Sept, 2017

\section{Key words:}

Blood purification

Capacity management

PICCO

\begin{abstract}
Objective: To explore the application value and nursing measures of pulse wave velocity dilution combined with continuous cardiac output measurement (PICCO) and trans arterial waveform analysis (Eduardo Vigileo) in continuous renal replacement therapy (CRRT) capacity management. Methods: 60 patients undergoing acute renal injury were divide into two groups, 30 randomly selected patients using PICCO for monitoring and the other 30 were monitored by Vigileo method. From the CI, SVR, SVV and other aspects of capacity management in comparison with the correlation between the two. Results: There were three correlations between CI, SVR and SVV. Conclusion: PICCO and Vigileo method have significant different in the capacity monitoring and management of patients with acute renal injury $(\mathrm{P}>0.05)$. However, due to the measurement of parameters in PICCO technique, shrink function and hemodynamic parameters of a more comprehensive reflection. And the only flow of micro-trauma, do not inject ice water to maintain the pipeline closed, reduce the infection is a major feature.
\end{abstract}

\section{Materials and methods}

\subsection{General information}

A total number of 60 patients with acute renal injury were selected from June 2013 to June 2015, and the diagnostic criteria were consistent with AKI. Including 35 cases of male and 25 cases of the female; aged 27 to 81 years, all cases were treated with CRRT, 30 patients were randomly selected from 60 patients to use PICCO monitoring guidance, and the other 30 with the only flow monitoring. There were no statistically significant differences in age, sex, and condition between the two groups.

\subsection{PICCO monitoring method}

The femoral artery was inserted into the femoral artery to dilute the catheter and the catheter was connected to the PICCO monitor. The indicator was rapidly injected into the central venous catheter with $10-15 \mathrm{ml}$ of ice saline, which was repeated three times to remove the mean. To get: Cardiac output index (CI), stroke volume variability (SVV), intrathoracic blood volume (ITBV), extra-vascular lung water (EVLW), systemic vascular resistance index (SVRI) and Global end diastolic volume (GEDV), and so on ${ }^{[2]}$.

\subsection{Vigileo monitoring methods}

The pedicle arterial puncture pathway was estab-lished and the waveform of the peripheral arteries was continuously analyzed after the sensor was connected to monitor cardiac output (CO), stroke volume (SV), peripheral vascular resistance index (SVRI), stroke volume variability (SVV), and other parameters to reflect the blood volume status.

\subsection{Statistical methods}

All the measurement data should be expressed by the standard deviation of \pm . The data were analyzed using SPSS 19.0, and the comparison between the two groups 
was carried out. Two different test results were calculated. And there is a certain statistical significance, that is, $\mathrm{P}<$ 0.05 .

\section{Test results}

Two monitoring methods to guide CRRT treatment, three days after the CI, SVR, SVV before and after comparison. The first day is: $2.7 \pm 1.1 ; 1154.36 \pm 213.76 ; 12.6 \pm 4.8$. The third day is: $3.6 \pm 0.5 ; 2037.64 \pm 64.67 ; 10.3 \pm 2.6$. The first day of the Vigileo is $2.9 \pm 0.8 ; 1281.36 \pm 316.68$; $13.4 \pm 4.4$. The third day is: $3.4 \pm 0.5 ; 1978.55 \pm 84.96$; $11.3 \pm 2.1$.

As can be seen from the table, both PICCO and Vigileo monitoring, 3 days after the data were stable, no significant difference between the two $(\mathrm{P}>0.05)$. But the first day and the third day of monitoring, the data were significantly different $(\mathrm{P}<0.05)$. Two groups of patients, where 22,20 people were using norepinephrine, so each group according to the random number of each order 20 people were the average pressure $<65 \mathrm{mmHg}$, lactic acid $\geq 4 \mathrm{mmol} / \mathrm{L}$ incidence, the amount of norepineph-rine several aspects of comparison It can be seen that the use of norepinephrine in the use of CRRT process, PICCO monitoring guidance capacity monitoring in the average pressure, the incidence of high lactic acid was significantly reduced, the amount of norepinephrine smaller.

\section{Discussion}

CI, SVRI, SVV is the power parameter of the blood flow that PICCO and VIC can collect together. In this, an important indicator of Vigileo monitoring system is SVV. SVV can reflect the preload state of the patient well out, and effectively eliminate the body's myocardial compliance and specific chest pressure on the impact of pressure indicators. At the same time, as one of the important indicators of Vigileo monitoring system SVV can correctly predict the human cardiovascular system to reflect the effect of liquid load, and thus more timely and effective, scientific and rational response to the treatment of liquid, is functional blood, one of the important indicators of flow dynamics monitoring ${ }^{[3]}$. Experiments show that there is no significant difference in the capacity monitoring between PICCO group and CDC group. However, PICCO technique has more measurement parameters, which can reflect the changes of hemodynamic parameters and cardiac systolic and diastolic function comprehensively, such as ITBV. The related indexes of circulating blood volume in critically ill patients. The specific capacity of the heart of the human body and the degree of myocardial compliance with the capacity of the parameters to reflect its elimination, you can more accurately the body's specific capacity of the heart load response. And EVLW, which is related to the capacity of the liquid, that is to say, that it can be applied to the monitoring of liquid capacity in human diseases. At the same time, the judgment of pulmonary edema is the only bedside quantitative, Sexual and sensitivity to a large extent than blood gas analysis and X-ray to better. So the application of CRRT in patients with cardiac dysfunction is broader. While the only stream to monitor the micro-trauma, do not inject ice water to maintain the tightness of the pipeline to reduce the infection is a major feature of it.

\section{References}

[1] Fu Liqin, Han Fang, Yao Huiping. PICCO Monitoring in Patients with Severe Infection CRRT Capacity Management and Nursing Measures, Chinese Journal of Infectious Diseases, 2012, 22(12): 2528-2530.

[2] Wang Zhi, Liu Ye, Tang Wei Bing. Vigileo Monitoring System in Patients with Traumatic Shock Mechanical Ventilation in the Application of the Value of Recovery, Guangdong Medical, 2014, 10(19): 3069-3071. [3] Mongegarc Ami, Gilcanoa, Raciaromerom. Dynamic Arterial Elastane to Predict Arterial Pressure Response to Volume Loading in Preload-Dependent Patients. CritCare, 2011, 15(1): R15. 